\title{
THE DOUBLE SIX OF LINES AND A THEOREM IN EUCLIDEAN PLANE GEOMETRY
}

\author{
by JOHN DOUGALL
}

(Received 5th June, 1950)

The object of the present paper is to establish the equivalence of the well-known theorem of the double-six of lines in projective space of three dimensions and a certain theorem in Euclidean plane geometry. The latter theorem is of considerable interest in itself for two reasons. In the first place, it is a natural extension of Euler's classical theorem connecting the radii of the circumscribed and the inscribed (or the escribed) circles of a triangle with the distance between their centres. Secondly, it gives in a geometrical form the invariant relation between the circle circumscribed to a triangle and a conic inscribed in the triangle. For a statement of the theorem, see $\S 13(4)$.

1. The configuration of the double-six of lines was discovered by the Swiss mathematician Schläfli in connection with the theory of the twenty-seven lines on a cubic surface.

Schläfli showed that from the twenty-seven lines, two sets of six lines, say

$$
\begin{aligned}
& a, b, c, d, e, f ; \\
& a^{\prime}, b^{\prime}, c^{\prime}, d^{\prime}, e^{\prime}, f^{\prime}
\end{aligned}
$$

could be selected (and that in thirty-six ways), with $a$ corresponding to $a^{\prime}, b$ to $b^{\prime}$, and so on, so that any line of either set of the double-six is met by the five which do not correspond to it in the other set; that is, $a^{\prime}$ meets $b, c, d, e, f ; a$ meets $b^{\prime}, c^{\prime}, d^{\prime}, e^{\prime}, f^{\prime}$; and so on.

2. Dropping all reference to a cubic surface, we have, in the above double-six, twelve lines and twelve examples of one line meeting five.

But only one theorem is involved; for the configuration may be built up as follows. Begin with any line $f$ and take any five lines meeting it, but with no pair of these meeting each other, viz., $a^{\prime}, b^{\prime}, c^{\prime}, d^{\prime}, e^{\prime}$. Any four of these is intersected by a second line besides $f$; we thus obtain $a, b, c, d, e$. The theorem is that $a, b, c, d, e$ are met by a twelfth line $f^{\prime}$; in other words, the two sets form a double-six.*

From the datum that no two of $a^{\prime}, b^{\prime}, c^{\prime}, d^{\prime}, e^{\prime}$ intersect, it is easy to prove that no two of $a, b, c, d, e, f$ intersect ; and that no two of $a^{\prime}, b^{\prime}, c^{\prime}, d^{\prime}, e^{\prime}, f^{\prime}$ intersect.

3. Any line in projective space of three dimensions can be specified by means of six homogeneous coordinates $p_{i},(i, j=1,2,3,4 ; i \neq j)$ defined in terms of the homogeneous coordinates $\left(x_{1}, x_{2}, x_{3}, x_{4}\right)$ and $\left(y_{1}, y_{2}, y_{3}, y_{4}\right)$ of any two points in it by the equations

$$
p_{i j}=x_{i} y_{j}-x_{j} y_{i} \text {. }
$$

These six coordinates $p_{i j}$ are connected by the homogeneous equation of the second degree

$$
p_{12} p_{34}+p_{13} p_{42}+p_{14} p_{23}=0 \text {. }
$$

It follows that the lines of projective space of three dimensions may be represented by the points of a quadric $Q$ in projective space of five dimensions.

Our main concern is with the condition that two lines should intersect. This condition is in effect that the two points on $Q$ which represent the lines are conjugate with respect to $Q$.

* Salmon, Analytic Geometry of Three Dimensions, Vol. II (5th edition) $\$ \S 534$, 536a. H. F. Baker, Principles of Geometry, Vol. III, p. 159 ; Vol. IV, pp. 58-64. 
In fivefold space, instead of a tangent plane to a surface we have a tangent fourfold and the condition that two points on $Q$ are conjugate with respect to $Q$ is that the tangent fourfold at either passes through the other; or again, that the line joining the points lies entirely on $Q$. Thus, using the same symbols to denote the lines of $\S 1$ and the points which represent them on $Q$, the fourfold $b c$ def is the tangent fourfold at $a^{\prime}$; acdef is the tangent fourfold at $b^{\prime}$; and so on.

The theorem of the double-six can now be stated in the equivalent form :

If $a, b, c, d, e, f$ are six points on $Q$, and if $b c d e f$, acdef, abdef, abcef, abcdf are tangent fourfolds, then abcde is also a tangent fourfold.

4. By means of the change of coordinates

$$
\begin{aligned}
& p_{12}=x+i w, p_{13}=y+i u, p_{14}=z+i t, \\
& p_{34}=x-i w, p_{42}=y-i u, p_{23}=z-i t,
\end{aligned}
$$

in the five-dimensional space in which $Q$ lies, the equation of $Q$ is brought to the form

$$
x^{2}+y^{2}+z^{2}+w^{2}+t^{2}+u^{2}=0,
$$

By homogeneous linear transformations which leave the quadratic form

$$
x^{2}+y^{2}+z^{2}+w^{2}+t^{2}+u^{2}
$$

invariant, other coordinate systems can be found in which the equation of $Q$ takes the same form. Among these there are systems for which any given fourfold is one of the coordinate fourfolds, say the fourfold $u=0$. Furthermore, since there exist fourfolds which do not pass through any one of a particular set of six given points, it is possible to choose a coordinate system such that $Q$ has the form (1) and the coordinate fourfold $u=0$ does not pass through any of the six points $a, b, \ldots, f$. Let us choose such a system of coordinates and then introduce non-homogeneous coordinates by replacing the ratios of $x, y, z, w, t$ to $u$ by $i x, i y, i z$, $i w$ and it respectively, so that, in the non-homogeneous coordinate system, $Q$ has equation

$$
x^{2}+y^{2}+z^{2}+w^{2}+t^{2}=1
$$

From now on we shall only use, in the five-dimensional space in which $Q$ lies, coordinate systems obtained from this system by orthogonal transformations. We shall introduce the terminology of metric geometry into this space by calling the function

$$
\left(x_{1}-x_{2}\right)^{2}+\left(y_{1}-y_{2}\right)^{2}+\ldots+\left(t_{1}-t_{2}\right)^{2} \text {, }
$$

in any one of these coordinate systems, and therefore in all of them, the square of the distance between the points $\left(x_{1}, y_{1}, \ldots, t_{1}\right)$ and $\left(x_{2}, y_{2}, \ldots, t_{2}\right)$ and referring to the coordinate systems as rectangular cartesian coordinate systems.

When this has been done the quadric $Q$ becomes a hypersphere, or as we shall call it to indicate its dimensions, a five-sphere in the, now complex Euclidean, five-dimensional space. It is the five-sphere with centre at the origin and radius unity.

5. The point $f(\S 1)$ can be taken to be the point $(0,0,0,0,1)$. For if it is not initially this point, a new rectangular cartesian coordinate system can be introduced in which it is. (Compare with the ordinary sphere $x^{2}+y^{2}+z^{2}=a^{2}$ ).

The equation of the tangent fourfold to $Q$ at the point $\left(x_{1}, y_{1}, z_{1}, w_{1}, t_{1}\right)$ is

$$
x x_{1}+y y_{1}+z z_{1}+w w_{1}+t t_{1}=1 \text {. }
$$

Let this pass through $f$; then $t_{1}=1$. But

therefore

$$
x_{1}^{2}+y_{1}^{2}+z_{1}^{2}+w_{1}^{2}+t_{1}^{2}=1
$$

$$
x_{1}^{2}+y_{1}^{2}+z_{1}^{2}+w_{1}^{2}=0 \text {, }
$$

a relation which is fundamental for what follows. 
6. As a first step towards reaching a plane figure, we now project the figure on $Q$ from $f$ onto the fourfold $t=t_{0}$ where $t_{0}$ is a constant. This fourfold we shall refer to as $S_{4}$.

The five relations given and the one to be proved, as stated in $\S 3$, have now to be transferred to the figure in $S_{4}$.

Let $a, b, c, d$, e project into $A, B, C, D, E$. The points $B, C, D, E$ lie on two fourfolds, viz., on the tangent fourfold at $a^{\prime}(b c d e f)$ and on $t=t_{0}$. Hence $B, C, D, E$ lie on a threefold in the fourfold $t=t_{0}$; in this fourfold the variable coordinates are $x, y, z, w$.

If $a^{\prime}$ is the point $\left(x_{1}, y_{1}, z_{1}, w_{1}, t_{1}\right)$, the equation of this threefold in terms of these coordinates is, from $\S 5(1)$, since $t=t_{0}$ and $t_{1}=1$,

where, by $\S 5(2)$,

$$
x x_{1}+y y_{1}+z z_{1}+w w_{1}=1-t_{0}
$$

$$
x_{1}^{2}+y_{1}^{2}+z_{1}^{2}+w_{1}^{2}=0 \text {. }
$$

Any threefold (in any $S_{4}$, i.e., in any fourfold whose equation in some rectangular cartesian coordinate system is $t=t_{0}$ ) whose equation is of the form

where

$$
l x+m y+n z+p w=q,
$$

$$
l^{2}+m^{2}+n^{2}+p^{2}=0
$$

we shall call a special threefold.

Thus, in virtue of (1) and (2), $B C D E$ is a special threefold ; and so also, similarly, are the threefolds $A C D E, A B D E, A B C E$ and $A B C D$.

7. To make certain that the definition is consistent, it is necessary to verify that, if the condition for a special threefold is satisfied in one rectangular cartesian system, it is true in

\begin{tabular}{|c|c|c|c|c|}
\hline & $x$ & $y$ & $z$ & $w$ \\
\hline$X$ & $l_{1}$ & $m_{1}$ & $n_{1}$ & $p_{1}$ \\
\hline$Y$ & $l_{2}$ & $m_{\mathbf{2}}$ & $n_{2}$ & $p_{\mathbf{2}}$ \\
\hline$Z$ & $l_{3}$ & $m_{3}$ & $n_{3}$ & $p_{3}$ \\
\hline$W$ & $l_{4}$ & $m_{\mathbf{4}}$ & $n_{4}$ & $p_{4}$ \\
\hline
\end{tabular}
all those for which the $S_{4}$ has equation $t=t_{0}$. For this and other reasons we consider the effect of the orthogonal transformation relating two such coordinate systems. This transformation is given by the scheme:

signifying that

$$
\begin{gathered}
X=l_{1} x+m_{1} y+n_{1} z+p_{1} w, \\
x=l_{1} X+l_{2} Y+l_{3} Z+l_{4} P
\end{gathered}
$$

and so on; where, since

$$
\begin{aligned}
& x^{2}+y^{2}+z^{2}+w^{2}=X^{2}+Y^{2}+Z^{2}+W^{2}, \\
& l_{1}^{2}+m_{1}^{2}+n_{1}^{2}+p_{1}^{2}=1 \text {, } \\
& l_{1}^{2}+l_{2}^{2}+l_{3}^{2}+l_{4}^{2}=1, \\
& l_{1} l_{2}+m_{1} m_{2}+n_{1} n_{2}+p_{1} p_{2}=0 \text {, } \\
& l_{1} m_{1}+l_{2} m_{2}+l_{3} m_{3}+l_{4} m_{4}=0 \text {, }
\end{aligned}
$$

and so on.

We note that if

then

$$
l x+m y+n z+p w=L X+M Y+N Z+P W,
$$

$$
l^{2}+m^{2}+n^{2}+p^{2}=L^{2}+M^{2}+N^{2}+P^{2},
$$


so that $l^{2}+m^{2}+n^{2}+p^{2}$, like $x^{2}+y^{2}+z^{2}+w^{2}$, is invariant under these changes of coordinates. It follows that the definition of special threefolds is consistent.

A comparison of (1) here and $\S 6$ (4) shows that a special threefold cannot be used as a coordinate threefold.

We note also that for two points or two threefolds we have the following invariants :

$$
\begin{gathered}
x_{1} x_{2}+y_{1} y_{2}+z_{1} z_{2}+w_{1} w_{2}, \\
\left(x_{1}-x_{2}\right)^{2}+\left(y_{1}-y_{2}\right)^{2}+\left(z_{1}-z_{2}\right)^{2}+\left(w_{1}-w_{2}\right)^{2}, \\
l_{1} l_{2}+m_{1} m_{2}+n_{1} n_{2}+p_{1} p_{2} .
\end{gathered}
$$

8. In $S_{4}$, the locus with equation

$$
\left(x-x_{0}\right)^{2}+\left(y-y_{0}\right)^{2}+\left(z-z_{0}\right)^{2}+\left(w-w_{0}\right)^{2}=C^{2}
$$

is the four-sphere with centre $\left(x_{0}, y_{0}, z_{0}, w_{0}\right)$ and radius $C$.

The case where $C=0$ is of particular importance; the locus is then at once the foursphere of radius zero with centre $\left(x_{0}, y_{0}, z_{0}, w_{0}\right)$ and a four-cone, the asymptotic cone of the sphere (1).

The tangent fourfold to $Q$ at any point $g$ on it meets $Q$ in the four-sphere with centre $g$ and radius zero.

To prove this we need only, as we may, introduce in the five-dimensional space, a rectangular cartesian coordinate system in which $g$ has the coordinates given to $f$ in $\S 5$. The tangent fourfold at $g$ is then $t=1$, which meets $Q$ in points at which $x^{2}+y^{2}+z^{2}+w^{2}=0$, as required.

9. In the figure $A B C D E$ in $S_{4}$ we now have five data, viz., that the five threefolds $B C D E$, $A C D E, A B D E, A B C E$ and $A B C D$ are all special (\$ 6). These five data represent in $S_{4}$ the five data in the original five-dimensional space stated at the end of $\S 3$. We have now to consider the transform to $S_{4}$ of the conclusion from these data, stated at the same place, viz., that the fourfold abcde is a tangent fourfold to $Q$.

We assume that this conclusion is correct ; that is, we assume that the double-six theorem is true, and deduce from the tangency of abcde that $A, B, C, D, E$ lie on a four-sphere of radius zero.

We first form the equation of the four-cone with vertex at $f$ and base the section of $Q$ by the fourfold abcde. Let the equation of this fourfold be

$$
l x+m y+n z+p w+q t=r .
$$

If the origin is changed to $f$ by writing $t=1+\tau$, this equation becomes

$$
l x+m y+n z+p w+q \tau=r-q
$$

and the equation of $Q$ becomes

$$
x^{2}+y^{2}+z^{2}+w^{2}+\tau^{2}+2 \tau=0 .
$$

Hence, using the method familiar in three-dimensional space, the four-cone has equation

$$
x^{2}+y^{2}+z^{2}+w^{2}+\tau^{2}+2 \tau(l x+m y+n z+p w+q \tau) /(r-q)=0,
$$

and this meets $t=t_{0}$, i.e., $\tau=t_{0}-1$, or $\tau=h$, say, in the four-sphere

$$
x^{2}+y^{2}+z^{2}+w^{2}+h^{2}+2 h(l x+m y+n z+p w+q h) /(r-q)=0 .
$$

The square of its radius is

$$
\frac{h^{2}}{(r-q)^{2}}\left(l^{2}+m^{2}+n^{2}+p^{2}\right)-h^{2}-\frac{2 q h^{2}}{(r-q)}
$$


that is,

$$
\left\{h^{2} /(r-q)^{2}\right\}\left\{l^{2}+m^{2}+n^{2}+p^{2}-(r-q)^{2}-2 q(r-q)\right\} .
$$

The second factor of $(4)$ is

$$
l^{2}+m^{2}+n^{2}+p^{2}+q^{2}-r^{2}
$$

which is zero, since (1) is a tangent fourfold to $Q$.

It has therefore been proved, from the double-six theorem, that the four-sphere through $A, B, C, D, E$ has radius zero.

Thus, to return to the beginning of $\S 9$, it has been shown that the equivalent in $S_{4}$ of the double-six theorem is that, if the five threefolds $B C D E, A C D E, A B D E, A B C E$ and $A B C D$ are special (end of $\S 6$ ), then the four-sphere $A B C D E$ has radius zero, i.e., is a four-cone.

10. We now confine our attention to the four-dimensional space $S_{4}$ and particularly to the triangle $A B C$.

We choose, as origin of coordinates in $S_{4}$, the centre of the circle $A B C$ and axes such that the plane $A B C$ is the $x-y$ plane having equations $z=0, w=0$. This can be done in the following way. Take as $O x$ and $O y$ any two perpendicular lines through the origin $O$ and in the plane $A B C$; for $O z$ take any line in $S_{4}$ perpendicular to the plane $A B C$ and for $O w$ take either direction on the line in $S_{4}$ perpendicular to the lines $O x, O y, O z$.

In the coordinate system so chosen, let $D$ be the point $\left(x_{4}, y_{4}, z_{4}, w_{4}\right)$ and $E$ the point $\left(x_{5}, y_{5}, z_{5}, w_{5}\right)$, and let $D^{\prime}$ be the point $\left(x_{n}, y_{4}, 0,0\right)$ and $E^{\prime}$ the point $\left(x_{5}, y_{5}, 0,0\right)$. If the coordinate system is changed so that the plane $A B C$ remains the plane $z=0, w=0$, the $x$ and $y$ coordinates of $D^{\prime}$ will still be equal to those of $D$, and similarly with $E^{\prime}$ and $E$.

11. In the type of coordinate system just chosen, the threefold $A B C D$ has equation of the form

$$
\lambda z+\mu w=0,
$$

for it passes through the plane $z=0, w=0$. Since it is a special threefold,

$$
\lambda^{2}+\mu^{2}=0
$$

Hence $A B C D$ is either $z+i w=0$ or $z-i w=0$, and $A B C E$ is the other. It is merely a matter of notation to take $z+i w=0$ for $A B C D$ and $z-i w=0$ for $A B C E$. Then $D$ is the point $\left(x_{4}, y_{4}, z_{4}, i z_{4}\right)$ and $E$ is the point $\left(x_{5}, y_{5}, z_{5},-i z_{5}\right)(\S 10)$.

12. The remaining special threefolds $B C D E, A C D E$ and $A B D E$ all pass through $D$ and $E$.

Consider first $B C D E$. Let us choose the $x$ - and $y$-axes in the plane $A B C$ so that $B C$ has equation $y-y^{\prime}=0$. It may be remarked that this would not be possible if $b, c$ intersected; but it has been observed in $\S 2$ that they do not. The equation of $B C D E$ has then the form

$$
\lambda\left(y-y^{\prime}\right)+\mu z+n v=0,
$$

for it passes through the line $y-y^{\prime}=0, z=0, w=0$. Since the threefold is special,

Since it passes through $D$ and $E$,

$$
\lambda^{2}+\mu^{2}+\nu^{2}=0 \text {. }
$$

and

$$
\begin{aligned}
& \lambda\left(y_{4}-y^{\prime}\right)+(\mu+i \nu) z_{4}=0 \\
& \lambda\left(y_{5}-y^{\prime}\right)+(\mu-i \nu) z_{5}=0 ;
\end{aligned}
$$

hence $\lambda^{2}\left(y_{4}-y^{\prime}\right)\left(y_{5}-y^{\prime}\right)=\left(\mu^{2}+\nu^{2}\right) z_{4} z_{5}$, or, since $\lambda^{2}+\mu^{2}+\nu^{2}=0$,

$$
\left(y_{4}-y^{\prime}\right)\left(y_{5}-y^{\prime}\right)=-z_{4} z_{5}
$$

If $\left(\alpha_{4}, \beta_{4}, \gamma_{4}\right)$ and $\left(\alpha_{5}, \beta_{5}, \gamma_{5}\right)$ are the ordinary trilinear coordinates of $D^{\prime}$ and $E^{\prime},(1)$ gives

$$
\alpha_{4} \alpha_{5}=-z_{1} z_{5}
$$


Similarly,

and

$$
\begin{aligned}
& \beta_{4} \beta_{5}=-z_{4} z_{5} \\
& \gamma_{4} \gamma_{5}=-z_{4} z_{5} .
\end{aligned}
$$

$D^{\prime}$ and $E^{\prime}$ are therefore isogonal points of the triangle $A B C$. This property represents the data of the double-six theorem ( $(9)$.

If $k^{2}$ is the common value of $\alpha_{4} \alpha_{5}, \beta_{4} \beta_{5}$ and $\gamma_{4} \gamma_{5}$, then

$$
z_{4} z_{5}=-k^{2} \text {. }
$$

13. Next for the conclusion ( $(9)$ of that theorem, which was shown to be represented by the property that the four-sphere $A B C D E$ has zero radius.

What does this give in the triangle $A B C$ ?

The equation of the sphere $A B C D E$ has the form

$$
\left(x^{2}+y^{2}-R^{2}\right)+z^{2}+w^{2}+2 p z+2 q w=0,
$$

for it contains the circle whose equations are

$$
x^{2}+y^{2}-R^{2}=0, z=0, w=0,
$$

$R^{2}$ being the square of the radius of the circumcircle of the triangle $A B C$.

(i) The sphere (1) is to pass through the points $D\left(x_{4}, y_{4}, z_{4}, i z_{4}\right)$ and $E\left(x_{5}, y_{5}, z_{5},-i z_{5}\right)$. For both of these points $z^{2}+w^{2}=0$. Hence, from (1),

and

$$
\begin{aligned}
& x_{4}{ }^{2}+y_{4}{ }^{2}-R^{2}+2 z_{4}(p+i q)=0 \\
& x_{5}{ }^{2}+y_{5}{ }^{2}-R^{2}+2 z_{5}(p-i q)=0 .
\end{aligned}
$$

Thus

$$
\left(x_{4}{ }^{2}+y_{4}{ }^{2}-R^{2}\right)\left(x_{5}{ }^{2}+y_{5}{ }^{2}-R^{2}\right)=4 z_{4} z_{5}\left(p^{2}+q^{2}\right)
$$

from $§ 12(2)$.

$$
=-4 k^{2}\left(p^{2}+q^{2}\right) \text {, }
$$

(ii) The radius of (1) is zero; i.e.,

$$
p^{2}+q^{2}+R^{2}=0 .
$$

From (2) and (3),

$$
\left(x_{4}{ }^{2}+y_{4}{ }^{2}-R^{2}\right)\left(x_{5}{ }^{2}+y_{5}{ }^{2}-R^{2}\right)=4 k^{2} R^{2},
$$

that is to say, we have the theorem :

In a plane triangle, the product of the powers, with respect to the circumcircle, of two isogonal points is equal to $4 k^{2} R^{2}$; where, if $(\alpha, \beta, \gamma)$ and $\left(\alpha^{\prime}, \beta^{\prime}, \gamma^{\prime}\right)$ are the trilinear coordinates of the isogonal points, $k^{2}$ is the common value of $\alpha \alpha^{\prime}, \beta \beta^{\prime}$ and $\gamma \gamma^{\prime}$, and $R^{2}$ is the square of the radius of the circumcircle.

The preceding analysis can be regarded as a deduction of this theorem from the theorem of the double-six ( $\$ 3$, last sentence). But the analysis could be reversed, so the two theorems are equivalent.

Further, instead of thinking of $D^{\prime}$ and $E^{\prime}$ as isogonal points of the triangle, we may, in virtue of a fundamental focal property of conics, consider them to be the foci of a conic inscribed in the triangle, in which case $k^{2}$ is $b^{2}$, the square of the minor semi-axis.

If the conic is a circle, the theorem (4) at once reduces to the familiar theorem, due to Euler :

$$
\left(R^{2}-\delta^{2}\right)^{2}=4 R^{2} \rho^{2},
$$

where $\delta^{2}$ is the square of the distance between the circumcentre and the centre of the inscribed or of an escribed circle, and $\rho$ is the radius of the inscribed or escribed circle. 
14. An algebraic proof of the theorem of $\S 13$ (4) may be derived from the invariant theory of two conics.*

If the conics are

$$
\begin{aligned}
S & \equiv \frac{x^{2}}{a^{2}}+\frac{y^{2}}{b^{2}}-1, \\
S^{\prime} & \equiv(x-\alpha)^{2}+(y-\beta)^{2}-r^{2},
\end{aligned}
$$

the four invariants are :

$$
\begin{aligned}
& \Delta=-\frac{1}{a^{2} b^{2}}, \Theta=\frac{1}{a^{2} b^{2}}\left(\alpha^{2}+\beta^{2}-a^{2}-b^{2}-r^{2}\right), \\
& \Theta^{\prime}=\frac{\alpha^{2}}{a^{2}}+\frac{\beta^{2}}{b^{2}}-1-r^{2}\left(\frac{1}{a^{2}}+\frac{1}{b^{2}}\right), \Delta^{\prime}=-r^{2} .
\end{aligned}
$$

The origin being at the centre of the ellipse, the coordinates of the foci are $\pm a e, 0$ (where $a^{2} e^{2}=a^{2}-b^{2}$, and those of the centre of the circle are $\alpha, \beta$.

The condition that a triangle can be circumscribed to $S$ and inscribed in $S^{\prime}$ is :

$$
\Theta^{2}=4 \Delta \Theta^{\prime} \text {. }
$$

The expression of this formula in terms of $a, b, \alpha, \beta, r$ does not lend itself to immediate geometrical interpretation. We know, however, from the analysis in this paper that it must be equivalent to $\S 13(4)$, with $k^{2}=b^{2}$; that is, to

$$
\left\{(\alpha-a e)^{2}+\beta^{2}-r^{2}\right\}\left\{(\alpha+a e)^{2}+\beta^{2}-r^{2}\right\}=4 b^{2} r^{2} .
$$

The proof that (1) and (2) are the same is a matter of the simplest algebra.

* Salmon, Conic Sections, Chapter on Invariants and Covariants of Systems of Conics.

GLasgow 